\title{
First report of yellow fever virus in non-human primates in the State of Paraná, Brazil
}

\author{
Marcos Vinícius Tranquilin ${ }^{[1]}$, Ricardo Coelho Lehmkuhl[ ${ }^{[1]}$, Ângela Maron ${ }^{[2]}$, \\ Lineu Roberto da Silva ${ }^{[2]}$, Liane Ziliotto ${ }^{[1]}$, Meire Christina Seki ${ }^{[1]}$, \\ Gabriela Ronchi Salomon ${ }^{[3]}$, and Adriano de Oliveira Torres Carrasco ${ }^{[1]}$
}

[1]. Departamento de Medicina Veterinária, Universidade Estadual do Centro-Oeste, Guarapuava, PR. [2]. Centro de Informações e Respostas Estratégicas de Vigilância em Saúde, Secretaria de Estado da Saúde do Paraná, Curitiba, PR. [3]. Departamento de Biologia, Universidade Estadual do Centro-Oeste Guarapuava, PR.

\begin{abstract}
Sylvatic yellow fever is a zoonosis associated mainly with wild animals, especially those in the genus Alouatta, that act as the source of infection. Once infected, these animals pass the disease on to humans by way of an infected mosquito belonging to the genera Aedes, Haemagogus, or Sabethes. The present study is the first report of a case of yellow fever in non-human primates (NHP) in the State of Paraná, Brazil. After the case was diagnosed, several prophylactic measures were adopted to prevent outbreaks of the disease in humans.
\end{abstract}

Keywords: Arbovirus infections. Wild animals. Zoonosis.

\section{INTRODUCTION}

Virus-borne hemorrhagic fevers occur all over the world and are mostly caused by ribonucleic acid (RNA) viruses that belong to one of four families: Flaviviridae, Bunyaviridae, Arenaviridae, or Filoviridae. These are highly lethal zoonoses, and most of them are associated with wild animals, such as primates, rodents, and other mammals ${ }^{1}$.

The main representative virus of the Flaviviridae family is the yellow fever virus (YFV), which is a spherical virus with an envelope and two projections, namely the envelope (E) and membrane (M) proteins. YFV has a diameter of $50 \mathrm{~nm}$ and has a single strand of RNA with positive polarity ${ }^{1}$.

Yellow fever is an infectious, non-contagious disease transmitted to humans by the bite of an infected mosquito. The disease is considered endemic to countries of Central Africa and to several countries in Central and South America and has two different epidemiological cycles: an urban cycle and a sylvatic cycle. In the urban cycle, the disease is an anthroponosis, and there are no known animal reservoirs of epidemiological importance. Urban yellow fever has not been reported in Brazil since $1942^{2}$. In contrast, in the sylvatic cycle, which was the object of the present study, non-human primates, especially monkeys in the genera Alouatta, Cebus, Atelles, and Callithrix ${ }^{2,3}$,

Address to: Dr. Adriano de Oliveira Torres Carrasco. Dept ${ }^{\circ}$ Medicina Veterinária/ UNICENTRO. Rua Simeão Varella de Sá 03, 85035-040 Vila Carli, Guarapuava, PR, Brasil.

Phone: 5542 3629-8240

e-mail: adriano.carrasco@gmail.com

Received 16 May 2013

Accepted 2 July 2013 are the main source of infection, and the disease is a zoonosis transmitted by mosquitoes mainly in the genus Haemagogus.

Humans acquire sylvatic yellow fever when they enter the ecological niche of the arbovirosis, that is, forests (or regions close to them) in which these infected primates are found. The infected animals are bitten by mosquitoes that inhabit the canopy of the trees, and once infected, the mosquitoes transmit the disease to humans and other primates, which perpetuates the cycle of the disease ${ }^{1}$. It should be emphasized that primates (both human and non-human) function as amplifiers of the disease and not as reservoirs, as once they are infected, they either die, become cured, or in this case, become immune for the rest of their lives ${ }^{3}$.

New World primates are recognized as susceptible to YFV, and animals in the genus Alouatta are the most sensitive. These animals can be infected by small doses of YFV, whereas animals in the genus Cebus are highly resistant to YFV. In contrast, Old World primates are highly resistant to YFV and rarely demonstrate yellow fever ${ }^{3,4}$. The susceptibility of non-human primates and the identification of cases in these animals is the main indicator of the circulation of YFV in forest areas in the Americas ${ }^{3,4}$. Recently, human cases of sylvatic yellow fever in Brazil have been preceded by reports of the disease in animals ${ }^{5}$. Therefore, we could consider non-human primates as sentinels of YFV circulation in a given region, and epidemiological surveillance should be conducted in these animals ${ }^{4,5}$.

The improvement of this epidemiological surveillance could, to some extent, permit early detection of an outbreak and enable quick establishment of a transmission-blocking vaccination ${ }^{2}$. Vaccination with live vaccines containing strain 17D are still the most efficient method to prevent yellow fever and provide immunity for at least 10 years ${ }^{3,6}$.

The present study is the first report of a yellow fever case in a non-human primate in the State of Paraná, Brazil and discusses the public health implications of this case. 


\section{CASE REPORT}

At the beginning of 2008, one dead Alouatta caraya (black howler) was sent to the SAAS (Serviço de Atendimento a Animais Selvagens, Wild Animal Attention Service) of the Universidade Estadual do Centro Oeste (UNICENTRO). In particular, this non-human primate (NHP) was sent by the Environmental Police of Paraná, Salto Segredo platoon, after they responded to a call from employees of the hydroelectric plant located at Rio Bonito do Iguaçu (Figure 1) in the center-south region of the

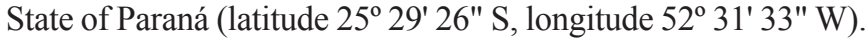

At the plant, the monkey was observed on the ground, disoriented and swaying, but still alive, and died a few hours later. At the moment the animal arrived, the CIEVS [Centro de Informações e Respostas Estratégicas de Vigilância em Saúde, Center for Information and Strategic Responses in Health Surveillance], a unit of the SESA [Secretaria de Estado da Saúde, State Health Office] of the State of Paraná was notified. The CIEVS authorized a post-mortem examination and approved the biosafety measures and instructions for collection of the material to be analyzed.

The physical evaluation revealed that the monkey was an adult, free-living male weighing approximately $7 \mathrm{~kg}$ that showed remarkable jaundice in the skin of the thorax

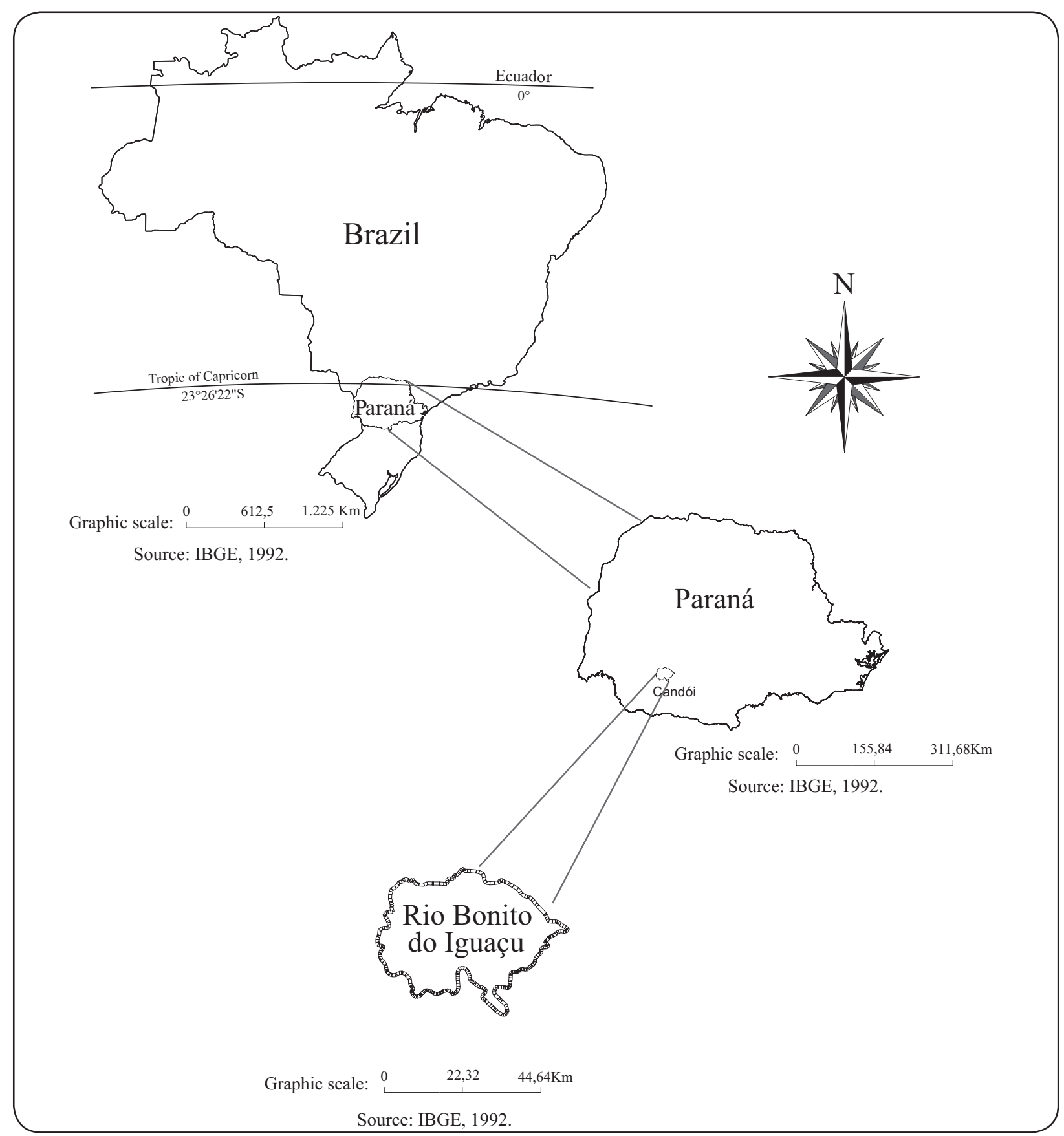

FIGURE 1 - Location of Rio do Bonito Iguaçu, State of Paraná, Brazil. 
and abdomen. The subcutaneous tissue showed intense jaundice, and large amounts of blood were present in the abdominal cavity; additionally, jaundice was present in the mesentery, liver, and peritoneum. The kidneys showed congestion caused by decubitus. The other organs did not show any noteworthy macroscopic changes. Samples of the liver, heart, lungs, kidneys, and brain were collected and sent to the Pathology Division of the Immunohistochemistry Laboratory of Instituto Adolfo Lutz for diagnosis of the yellow fever virus. Immunohistochemistry was conducted using an enzyme-conjugated polymer and secondary antibodies to detect YFV polyclonal antigens.

The assay for direct detection of YFV (immunohistochemistry) was positive only in the liver sample, which confirmed the first report of sylvatic yellow fever in a non-human primate in the State of Paraná. No other diagnostic techniques (virus culture or histopathological tests) were performed on the samples.

\section{DISCUSSION}

With a positive diagnosis of yellow fever, several prophylactic measures were adopted in the State of Paraná to prevent the occurrence of the disease in humans. Specifically, surveillance of free-living and captive primates in all rural and urban areas of the state was conducted, and the coverage of immunization in the state, mainly in the affected region, was expanded. These measures were implemented because cases in humans are usually preceded by reports of death in infected animals. Therefore, animals could serve as sentinels ${ }^{2,5}$ for the disease. Additionally, it is necessary to delimit the area of disease transmission based on environmental studies that evaluate the presence of vectors and reservoirs ${ }^{2}$.

After the positive detection of YFV in the animal, the State of Paraná changed, through the State Health Office (SESA), the risk area for the disease and increased the vaccination coverage in the state. Up until 2004, the vaccination area for YFV in the State of Paraná was restricted by its border with Paraguay. Based on the present case and the occurrence of more cases in humans in others regions of Paraná, by 2012 the recommended vaccination area comprised almost the entire state, excluding the region of the Paraná coast. Vaccinations are the main form of prevention of the disease and should immediately be established after the diagnosis of one positive case, either in humans or animals ${ }^{2,3}$, considering that one of the factors for the reemergence of yellow fever cases is the exposure of unvaccinated (susceptible) individuals to $\mathrm{YFV}^{6}$.

Monkeys in the genus Alouatta are important sentinels for YFV detection in a given region, as they are highly susceptible to $\mathrm{YFV}^{4,6}$. A similar finding was previously reported in the State of Rio Grande do Sul, where 173 monkeys in this genus were positive for YFV in laboratory tests in 2008 and $2009^{5}$ alone.
The immunohistochemistry methodology used in the present study is considered a standard technique, and is used in reference laboratories. One of the advantages of this method is that it allows the use of tissue samples from dead animals, which could aid the diagnosis of sentinels or animals that are highly susceptible to $\mathrm{YFV}^{3,5}$.

In Brazil, there have been periodic reports of yellow fever outbreaks. This periodic distribution of cases may be explained by the presence of vectors and the presence or circulation of infected non-human primates that act as virus amplifiers in the epidemiological cycle of the disease ${ }^{6}$. This circulation of infected monkeys, beyond migration, could occur through the illegal trade of wild animals, thus making it easier for the disease to enter an unaffected area where favorable conditions exist for the zoonosis to spread'.

These factors, in addition to the possibility that the infected monkey could have been bitten by hundreds of mosquitoes and consequently disseminated the YFV to a large number of susceptible individuals (both humans and monkeys) ${ }^{6}$, underscore the importance of constant surveillance of free-living and captive primates to effectively monitor the presence of YFV in these animals.

Based on the detection of this positive animal, a cooperation agreement was established between CIEVS/SESA and the SAAS [Wild Animal Attention Service] of UNICENTRO in Guarapuava, who agreed that live non-human primates would be kept in quarantine until a definitive diagnosis could be established. In addition, dead monkeys would also be sent to UNICENTRO for tissue collection and diagnosis. With these procedures, active surveillance is being conducted in the state, thus enabling the quick adoption of prophylactic measures to prevent outbreaks of the disease in humans and to determine the sites of occurrence of YFV in the State of Paraná.

\section{REFERENCES}

1. Figueiredo LTM. Febres hemorrágicas por vírus no Brasil. Rev Soc Bras Med Trop 2006; 39:203-210.

2. Tauil PL. Aspectos críticos do controle da febre amarela no Brasil. Rev Saude Publica 2010; 44:555-558.

3. Vasconcelos PFC. Febre amarela. Rev Soc Bras Med Trop 2003; 36:275-293.

4. Lima MA, Romano-Lieber NS, Duarte AMRC. Circulation of antibodies against yellow fever virus in a simian population in the area of Porto Primavera hydroelectric plant, São Paulo, Brazil. Rev Inst Med Trop 2010; 52:11-15.

5. Almeida MAB, Santos E, Cardoso JC, Fonseca DF, Noll CA, Silveira VR, et al. Yellow fever outbreak affecting Alouatta populations in Southern Brazil (Rio Grande do Sul). Ame J Primatol 2012; 74:68-76.

6. Vasconcelos PFC. Yellow fever in Brazil: thoughts and hypotheses on the emergence in previously free areas. Rev Saude Publica 2010; 44:1144-1149. 